\title{
Antiplasmin activity of electrophoretically separated human serum fractions
}

\author{
R. D. MANN, SUSAN COTTON, AND D. JACKSON \\ From the Clinical Research Department, Pfizer Ltd., Sandwich, Kent
}

SYNOPSIS The antiplasmin which migrates electrophoretically with the alpha $a_{2}$ globulins preponderates in effect over that of the alpha $a_{1}$ migrating antiplasmin. This preponderance persists at physiological $p \mathrm{H}$ value in vitro and the significance of this finding is discussed.

No evidence has been obtained of the existence of anti-urokinase activity in antiplasmin-free serum fractions.

The fibrinolytic activity of the enzyme plasmin is inhibited by two different plasma components which migrate electrophoretically with the alpha $a_{1}$ and alpha $_{2}$ globulins respectively. The alpha $a_{1}$ migrating antiplasmin combines relatively slowly and irreversibly with plasmin in a manner which is temperature dependent. The alpha $_{2}$ migrating antiplasmin produces an immediate, temperature-independent inhibition which is more readily reversible (Norman, $1958,1960)$. In addition, it has been suggested that antiplasmin activity resides largely in the alpha globulin fraction (Jacobbson, 1955).

Plasmin neutralization may, therefore, be predominantly due to an antiplasmin fraction which combines in a rapid and reversible manner with plasmin. As this concept is of importance, we have undertaken studies of the antiplasmin and antiurokinase activity of each fraction of normal human serum separated by curtain electrophoresis. In this paper, we report the antiplasmin activity of each such fraction, at the $p \mathrm{H}$ of the electrophoretic separation and at the physiological $p \mathrm{H}$ value, and the anti-urokinase activity of the fractions at the $p \mathrm{H}$ of separation, over a range of urokinase concentrations.

It has been found that, in this experimental system, the potency of the alpha $a_{2}$ antiplasmin preponderates over that of the alpha $\mathrm{a}_{1}$ antiplasmin at both $p \mathrm{H}$ values examined. We consider, therefore, that plasmin neutralization is likely to be predominantly rapid and reversible in the circumstances in which these characteristics are typical of the alpha $_{2}$ antiplasmin.

Received for publication 14 October 1965.
MATERIALS

The following were used:-Fibrinogen, human, grade $\mathbf{L}$ (bottles of 1 g.), Kabi Pharmaceuticals Ltd., plasminogen, human, grade B (120 casein units/vial), Kabi Pharmaceuticals Ltd; plasmin, human, grade B (28 casein units/ vial), Kabi Pharmaceuticals Ltd.; urokinase reference standard, human (2,400 Ploug units/vial), Leo Laboratories Ltd.; thrombin reagent, bovine (2,500 units/vial), Leo Laboratories Ltd.; veronal buffer $p \mathbf{H} 7 \cdot 4,0 \cdot 154 \mathrm{M}$.: sodium diethyl barbiturate 11.745 g., sodium chloride 14.67 g., hydrochloric acid $0.1 \mathrm{~N} 430 \mathrm{ml}$., distilled water to 2 litres; and Tris citrate buffer $p \mathrm{H} 8 \cdot 6: 0 \cdot 076 \mathrm{M}$. Tris $9.21 \mathrm{~g}$./litre, $0.005 \mathrm{M}$. citric acid $1.05 \mathrm{~g}$./litre.

\section{METHOD}

Fresh human serum was obtained on three separate occasions from a male subject without evidence of arterial disease or altered fibrinolytic activity and each specimen was individually separated by curtain electrophoresis into 47 fractions using Tris citrate buffer $p H$ 8.6. On each occasion, serum was applied at a rate of $38 \mathrm{ml}$./day. A potential of 2,100 volts was used for a period of 48 hours on two occasions and 24 hours during the third separation. A temperature of 5 to $7^{\circ} \mathrm{C}$. was maintained during the electrolysis with the buffer pump delivering $80 \mathrm{ml}$./hour.

The optical density of each fraction was determined at $280 \mathrm{~m} \mu$ using a Unicam SP.500 spectrophotometer. The fractions were of equal size and the $p H$ of each was determined using a direct reading $p \mathrm{H}$ meter (Electronics Instruments Ltd.).

Each fraction was then tested for interference with the plasmin-substrate interaction, the following reagents being added, in the order given, to assay tubes contained in an ice water bath at $4^{\circ} \mathrm{C}$. in which each reagent had been assembled.

1 Human fibrinogen, $0.6 \mathrm{ml}$. ( $5 \mathrm{mg}$ ), in veronal buffer pH $7 \cdot 4$ 
2 Serum fraction, $2 \mathrm{ml}$, or, in the control tubes, $2 \mathrm{ml}$. of the Tris citrate buffer used in the electrophoretic separation

3 Plasmin, $0.1 \mathrm{ml}$. (0.5 c.u.), in veronal buffer $p \mathrm{H} 7.4$

4 Calcium $(0.296 \mathrm{mg}$.) $0.2 \mathrm{ml}$., thrombin (5 units) mixture in veronal buffer $p \mathrm{H} 7 \cdot 4$.

The lysis time was commenced the moment the plasmin was added and the tubes were agitated as the calcium-thrombin addition was made. Each tube was then retained in the ice water bath for exactly five minutes to permit clotting to become complete, before the test clots and controls were transferred to a water bath at $37^{\circ} \mathrm{C}$. to permit lysis to occur.

The end point was recorded when air bubbles trapped in the gel were suddenly released and the opaque gel became totally transparent. In addition, the development of the sol phase was obvious when the tubes were inclined at an angle of $30^{\circ}$, and was associated with the development of a typical meniscus in place of the previously irregular gel surface.

On the third occasion, the fractions were concentrated by dialysis to double their previous strength and half their previous volume. The test of antiplasmin activity was then undertaken on each fraction both before and after careful titration to $p \mathrm{H} 7.4$ using $0.3 \mathrm{M}$ citric acid.

On the second occasion aliquots, each of $2 \mathrm{ml}$. serum fraction, were tested for anti-urokinase activity, the plasmin in the above experiments being replaced by the following reagents:-

1 Plasminogen, $0 \cdot 1 \mathrm{ml}$. (2.5 c.u.), in veronal buffer pH 7.4.

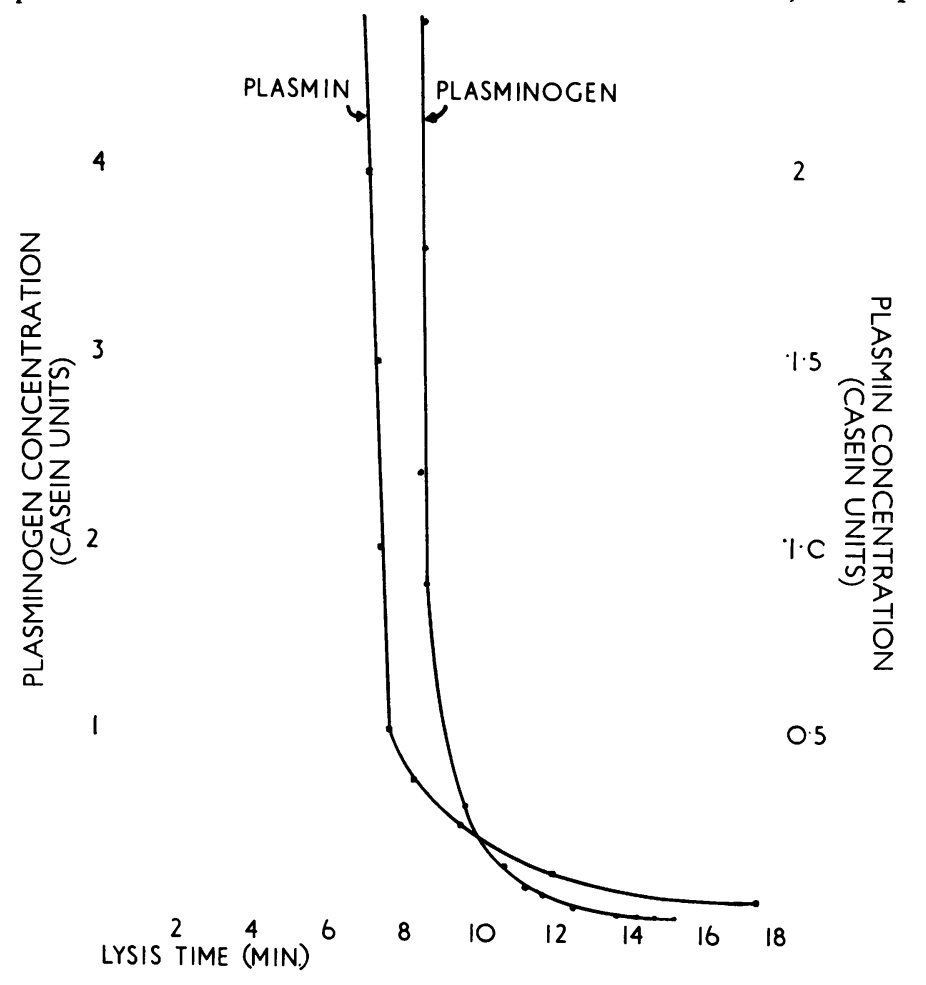

2 Serial concentrations of urokinase, $0 \cdot 1 \mathrm{ml}$., in veron

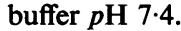

The experiments to detect anti-urokinase activity we undertaken with the consecutive serum fractions pooled in batches of four. Five clots were then prepared from each batch and the clots were individually activated wi log. concentrations of urokinase between the range $0.00 \mathrm{f}$ to 1.0 Ploug units. Controls were prepared as in the previous experiments.

The concentrations of plasminogen and plasmin use in these studies were derived from consideration of preliminary experiments in which they were found to optimal. Greater concentrations did not accelerafe further the rate of fibrinolysis and lesser concentration resulted in prolonged lysis times progressively dependept upon the increasing plasminogen or plasmin deficiency $\vec{\omega}$

\section{RESULTS}

EFFECT OF PLASMINOGEN AND PLASMIN CONCEN TRATION The results of the preliminary experiments to determine the effect of increasing plasminogen and plasmin concentrations in the experiments system used are illustrated in Figure 1. Concentrat tions of 2.5 c.u. plasminogen or 0.5 c.u. plasmin wege considered to be optimal.

ANTIPLASMIN ACTIVITY The antiplasmin effect, th is, the specific inhibitory effect of each seru

\section{FIG. 1. Serial concentrations of} plasminogen in a standard $2 \mathrm{ml}$. clot comprising $5 \mathrm{mg}$. human fibrinogen, human plasminogen, 200 Ploug units, urokinase, 0.296 mg. (0.001 M.) $\mathrm{CaCl}$ and 5 units bovine thrombin all in veronat buffer $\mathrm{pH}$ 7.4. Also serial concentrations of plasmin in a similar standard clot in which the plasminogen and urokinase were replaced by human plasmin. 


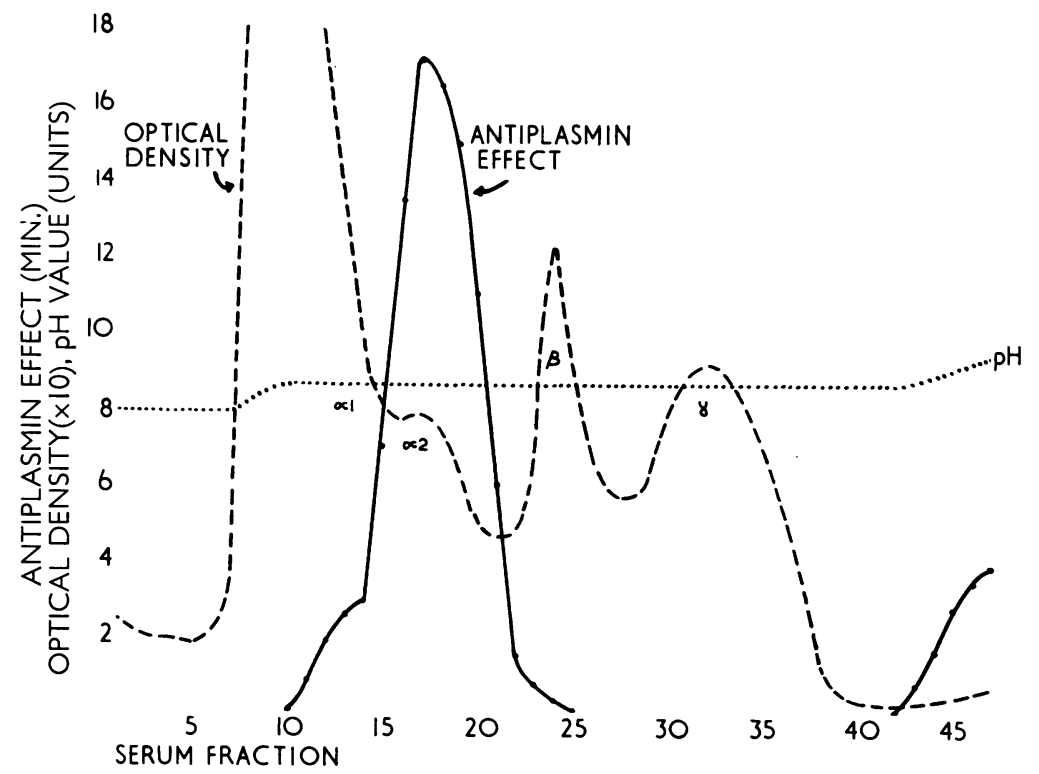

FIG. 2. The $\mathrm{pH}$ and optical density at $280 \mathrm{m \mu}$ of each fraction of human serum separated by curtain electrophoresis in Tris citrate buffer $\mathrm{pH} 8.6$; also antiplasmin effect, or interference with the plasmin-fibrin interaction of each fraction.

fraction on the plasmin-fibrin interaction, was calculated as the lysis time of the clot containing the serum fraction less the lysis time of the control clot.

The optical density, original $p \mathrm{H}$, and antiplasmin effect of each serum fraction are shown in Figure 2. Within the limits of minor experimental error, identical results were obtained on each of the three occasions and it will be observed that the potency of the alpha ${ }_{2}$ migrating antiplasmin greatly exceeded that of the alpha $a_{1}$ migrating antiplasmin.

The $p \mathrm{H}$ and antiplasmin effect of each serum fraction, after $50 \%$ concentration, both at the $p \mathrm{H}$ of separation and after titration to $p \mathrm{H} \mathrm{7.4}$ is shown in Fig. 3, from which it will be noted that the greater potency of the alpha $a_{2}$ antiplasmin persists at the physiological $p \mathrm{H}$ value. The potency of both antiplasmin fractions is greater at $p \mathrm{H} 8.6$ than at $p \mathrm{H} \mathrm{7.4}$.

From these graphs, it will be noted that an inhibitory effect on the plasmin-substrate interaction was detected in the post gamma-globulin region. This effect coincided with increasing alkalinity of these fractions in the original separation and was eliminated by titrating these fractions to $p \mathrm{H} 8.6$ as shown in Figure 3. It was also observed that this effect could be stimulated with buffers of increasing alkalinity up to and including $p \mathrm{H} 10$, and it is concluded, therefore, that the interference with the plasmin-substrate interaction in the post-gammaglobulin region is an artefact due to alkaline $p \mathrm{H}$ changes.

ANTI-UROKINASE EFFECTS When the plasmin in the above experiments, which were undertaken to detect antiplasmin activity, was replaced by plasminogen and urokinase, so that the experiments would also reveal anti-urokinase activity, it was found that no fraction exhibited an inhibitory effect which did not show antiplasmin activity.

The inhibitory activity of the fractions previously shown to exert an antiplasmin effect was demonstrated with each concentration of urokinase used. The inhibitory effect on the plasmin-substrate interaction, which was previously demonstrated in the post-gamma globulin region, was found to cause inhibition of urokinase-activated fibrinolysis when the urokinase concentration was 0.0001 Ploug units. This effect was less when the urokinase concentration was 0.001 Ploug units and the system was insensitive to this interference when greater concentrations of urokinase were used.

The possibility remains that an anti-activator, if present in the fractions which inhibited the lysis of fibrin by plasmin, would be masked by this specific antiplasmin effect.

\section{DISCUSSION}

Jacobbson (1955), using filter-paper zone electrophoresis, found human serum to contain two trypsin inhibitors which migrated with the alpha and alpha $a_{2}$ globulin fractions respectively; the alpha $_{2}$ fraction inhibited both plasmin and trypsin while the alpha $a_{1}$ fraction inhibited only trypsin. Norman (1958) and Norman and Hill (1958), using 


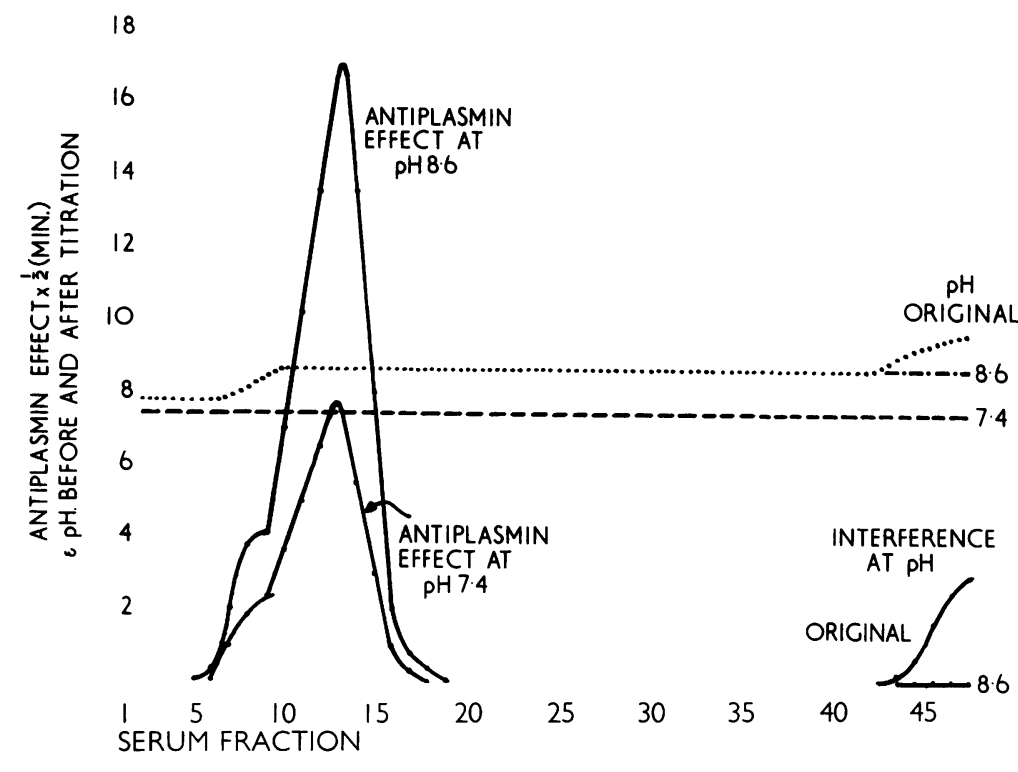

FIG. 3. The $\mathrm{pH}$ and antiplasmir effect of each fraction of human serum before and after titration to $\mathrm{pH}$ 7.4. The interference with 0 the plasmin-substrate interaction originally present in the postgamma-globulin region, and associated with increasing alkalinity of these fractions, was eliminated by an intermediate titration to $\mathrm{pH} 8 \cdot 6$.

starch zone electrophoresis, reported one antiplasmin migrating with the alpha ${ }_{1}$ globulins and another migrating with the alpha $a_{2}$ globulins.

Rybak and Rejnek (1959) studied the antiplasmin activity of plasma fractions, separated by preparative electrophoresis, ethanol fractionation, and fractionation by metal salts, and found activity to be restricted to the alpha-globulins. Nilsson, Krook, Sternby, Söderberg, and Söderström (1961), using preparative paper electrophoresis, found that in normal serum antiplasmin activity was mainly recovered from the alpha ${ }_{2}$ globulin fraction, whereas most of the antitrypsin activity existed in the alpha globulin region. Finally, Riding and Ellis (1964) confirmed the previous observations of Norman and Hill (1958) that antiplasmin activity is found in the Cohn ethanol fractions IV-4 and IV-1, corresponding to the alpha $a_{1}$ and alpha ${ }_{2}$ migrating antiplasmins. In addition, these authors found inhibitory activity in fraction III-O, the lipoprotein-containing fraction of human plasma.

In our own experiments, it has been found that the potency of the alpha $\mathrm{a}_{2}$ antiplasmin consistently preponderates over that of the alpha $a_{1}$ antiplasmin and this effect persists at physiological $p \mathrm{H}$ range. As the alpha $_{2}$ antiplasmin neutralizes plasmin in a rapid and reversible manner (Norman, 1960), it appears reasonable to infer that plasmin neutralization is predominantly rapid and reversible in nature.

It has been shown that fibrin can actually partly reverse the combination of plasmin with the alpha antiplasmin (Ambrus, Back, and Ambrus, 1960) and that an increase in the concentration of the protein substrate can readily reverse the neutralization due to the alpha ${ }_{2}$ antiplasmin (Norman, 1960).

These considerations explain how a fibrin clof can be lysed by plasmin even in the presence of potent inhibitors, and Ambrus and Markus (196. have suggested that plasmin-antiplasmin comples exists as a reservoir of fibrinolytic enzyme which dissociates in the presence of fibrin. In addition, $\overrightarrow{\vec{B}}$ has been demonstrated that, when the plasmin concentration is increased in a plasmin-fibrin inhibitor system, a portion of the inhibitor which was previously free becomes involved in the reaction (Rybak, 1962). However, increasing concentrations of the inhibitor do not completely inactivate plasmin, part at least of which can be made available to lyse fibrin (Taylor, Allen, and Bickford, 1964).

The concept that plasmin-antiplasmin complex may form a circulating reservoir of plasmin which is innocuous to other circulating proteins but whic forms a ready source of plasmin when this is needed for the lysis of fibrin is, therefore, of interest. Tho extent of such plasmin binding may be appreciated from the work of Nanninga and Guest (1964) whs have shown that bound plasmin is present in hundred-fold greater concentration than free plasmtr at plasmin concentrations below $10^{-7} \mathrm{M}$. The faci that a failure of fibrinolysis may occur in the presence of high levels of inhibitory activity is relevant and has been established by Maki ang Nagasawa (1963).

Clearly, thrombolysis is dependent upon activatio of plasminogen adsorbed on to the surface of fibrif (Fearnley, 1953, 1961; Sherry, Fletcher, an\& 
Alkjaersig, 1959b). However, the presence of the fibrin polymer is not a physiological event and it may be that, in the physiological functioning of the fibrinolytic system, plasmin exerts an indirect effect by the repletion of a predominantly dynamic plasmin-antiplasmin complex or equilibrium.

Finally, it may be noted that the experimental studies reported have shown no evidence of antiurokinase activity in antiplasmin-free serum fractions, although this evidence does not exclude the possibility that specific anti-activator activity may exist in serum fractions which inhibit the lysis of fibrin by plasmin.

We are grateful to the Board of Pfizer Limited for permission to publish this paper and for meeting the expense of this investigation.

The curtain electrophoresis of the serum used in these experiments was undertaken by Dr. T. Freeman, member of the staff of the Medical Research Council, National Institute of Medical Research, Mill Hill, and we wish to express our sincere thanks for his help and advice.

\section{REFERENCES}

Ambrus, C. M., Back, N., and Ambrus, J. L. (1960). Fed. Proc., 19, 58. and Markus, G. (1960). Amer. J. Physiol., 199, 491.

Fearnley, G. R. (1953). Nature (Lond.), 172, 544.

(1961). Lancet, 1, 992.

Jacobbson, K. (1955). Scand. J. clin. Lab. Invest., suppl., 14, 91.

Maki, M., and Nagasawa, K. (1963). Tohoku J. exp. Med., 78, 363.

Nanninga, L. B., and Guest, M. M. (1964). Arch. Biochem., 108, 542.

Nilsson, I. M., Krook, H., Sternby, N. H., Söderberg, E., and Söderström, N. (1961). Acta med. scand., 169, 323.

Norman, P. S. (1958). J. exp. Med., 108, 53.

(1960). Amer. J. Cardiol., 6, 390.

- and Hill, B. M. (1958). J. exp. Med., 108, 639.

Riding, I. M., and Ellis, D. (1964). J. Atheroscler. Res., 4, 189.

Rybak, M. (1962). Physiol. bohemoslov., 11, 470.

and Rejnek, J. (1959). Clin. chim. Acta, 4, 364.

Sherry, S., Fletcher, A. P., and Alkjaersig, N. (1959b). Physiol. Rev., $39,343$.

Taylor, F. B., Jr., Allen, L. W., and Bickford, A. F., Jr. (1964). Arch. Biochem., 104, 277. 